\title{
27-day variation in cloud amount in the Western Pacific warm pool region and relationship to the solar cycle
}

\author{
Y. Takahashi ${ }^{1}$, Y. Okazaki ${ }^{2}$, M. Sato ${ }^{1}$, H. Miyahara ${ }^{3}$, K. Sakanoi ${ }^{4}$, P. K. Hong ${ }^{5}$, and N. Hoshino ${ }^{2}$ \\ ${ }^{1}$ Department of Cosmosciences, Hokkaido University, Hokkaido, Japan \\ ${ }^{2}$ Department of Geophysics, Tohoku University, Tohoku, Japan \\ ${ }^{3}$ Instuitute for Cosmic Ray Research, The University of Tokyo, Tokyo, Japan \\ ${ }^{4}$ Department of Natural Sciences, Komazawa University, Komazawa, Japan \\ ${ }^{5}$ Department of Complexity Science and Engineering, The University of Tokyo, Tokyo, Japan
}

Received: 5 April 2009 - Published in Atmos. Chem. Phys. Discuss.: 16 July 2009

Revised: 23 December 2009 - Accepted: 11 January 2010 - Published: 15 February 2010

\begin{abstract}
Although linkages between solar activity and the earth's climate have been suggested and the 11-year cycle in solar activity evident in sunspot numbers is the most examined example of periodicity in previous studies, no quantitative evidence indicating a relationship for tropospheric phenomena has been found for a short period. Based on FFT analysis for OLR (Outgoing Longwave Radiation) compared with the F10.7 index, we clearly demonstrate a 27-day variation in the cloud amount in the region of the Western Pacific warm pool, which is only seen in the maximum years of 11year solar activity. The average spectrum in such years also shows an enhancement in the range of the MJO (MaddenJulian Oscillation) period. Although there exist some explanations for possible mechanisms, the exact cause is unknown. Therefore, the proposed connection between 27-day cloud variation and solar cycle in the WPWP region is still a hypothesis and various kinds of varification based on other meteorological and solar parameters are strongly required.
\end{abstract}

\section{Introduction}

Linkages between solar activity and the earth's climate have been suggested in previous studies. The 11-year cycle in solar activity evident in sunspot numbers is the most examined example of periodicity, and it is clearly recognized in variations in the thermal structure and dynamical motion of the

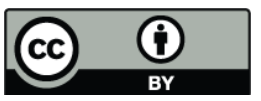

Correspondence to: Y. Takahashi (yukihiro@ep.sci.hokudai.ac.jp) stratospheric atmosphere (Haigh, 1994; Kodera, 1995; Shindell et al., 1999). Synchronization between the flux of galactic cosmic rays and cloud amount has also been reported in terms of the solar cycle (Svensmark, 1998). In a subsequent paper by Marsh and Svensmark (2003), the proposed effect is attributed to low-level clouds. Also in the longer time scale, the synchronization between the cosmic rays and cloud amount is discussed by Miyahara (2008). But it should be noted that there exist some sceptical standpoints toward the solar effect, such as Damon and Laut (2004). Variations in the stratosphere related to the period of apparent solar rotation have also been suggested (Nastrom and Belmont, 1978; Hood, 1984); however, for such a short period, no quantitative evidence exists to indicate a relationship between solar activity and tropospheric phenomena. Here we focus on cloud amount variation with $\sim 1$ month periodicity using Outgoing Longwave Radiation (OLR) and examine the modulation of the periodicity, paying attention to 11-year solar cycle.

\section{Analysis and results}

We used OLR data as a proxy for the cloud amount (Liebmann and Smith, 1996). The OLR is the total amount of thermal radiation emitted from the earth to interplanetary space. Low values of OLR at low latitudes are associated with clouds, as radiation from the low-temperature cloud top is weaker than that from the surface. That is, OLR is an indicator of the cloud amount. Figure 1 shows the map of the spectral power of OLR in the period of 25-29 day, that is

Published by Copernicus Publications on behalf of the European Geosciences Union. 


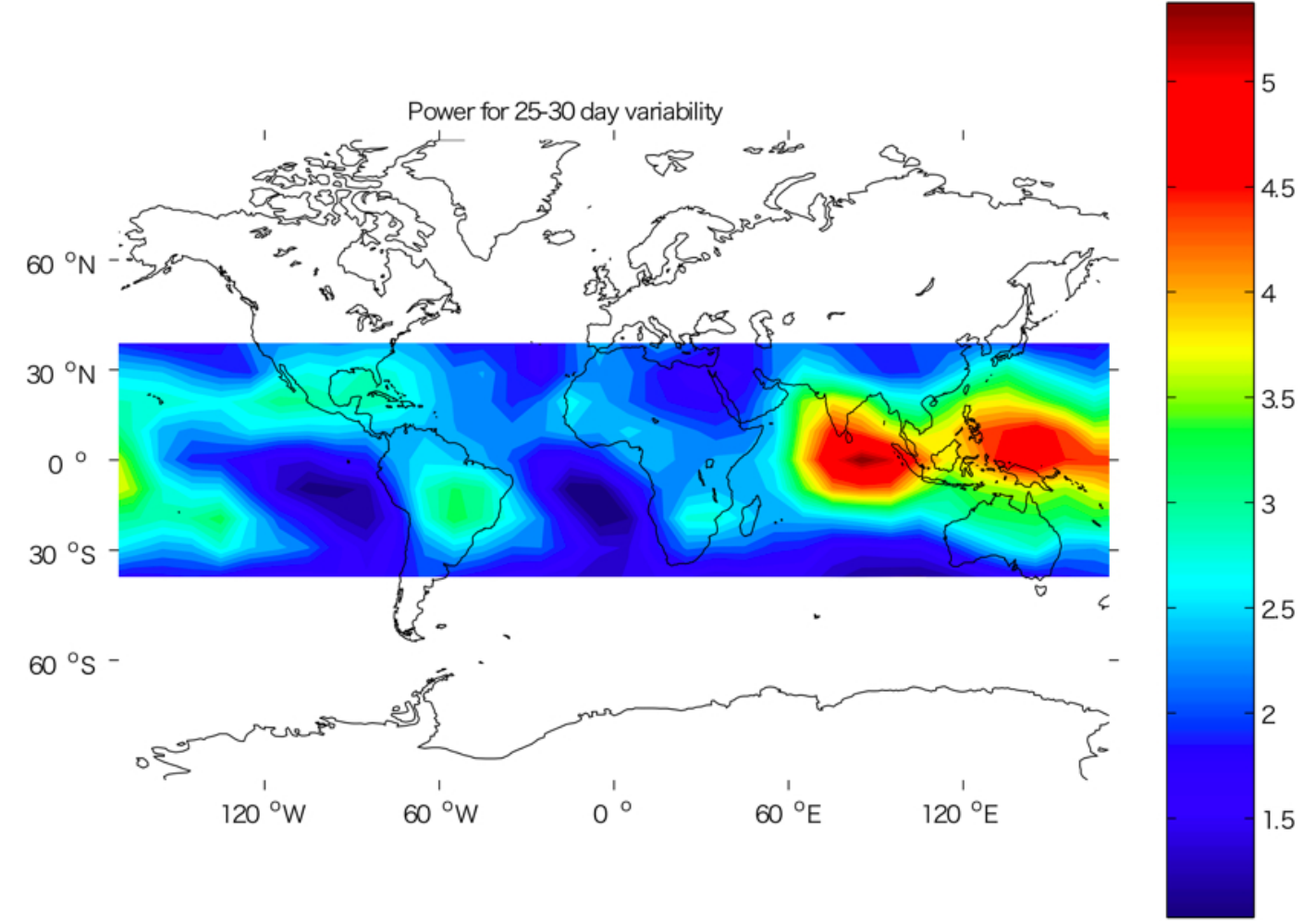

Fig. 1. Map of the spectral power of OLR in the period of 25-29 day.

about the cycle of solar rotation (27 days), for the low latitude region. Apparently two regions, that is, the eastern part of the Indian Ocean and the central part of the Western Pacific Warm Pool (WPWP) centred at $140^{\circ} \mathrm{E}, 10^{\circ} \mathrm{N}$, show significant enhancement. The WPWP, which shows the broader enhanced area in the power spectrum, is known to show the highest sea surface temperature in any ocean and strong atmospheric convective activity. In the present study, as the first step, we conducted a spectral analysis for WPWP area to investigate this periodicity in detail for the OLR variation.

The OLR was spatially integrated over the longitudes $130^{\circ}$ to $150^{\circ}$ and the latitudes $0^{\circ}$ to $10^{\circ}\left(2.5^{\circ}\right.$-square grid $)$. The period of analysis is from 1980 to 2003 , covering almost two and a half 11-year solar cycles. After removing longterm variations using a high-pass filter (cut-off period of 180 days), we calculated the power spectra of individual yearly data using the Fourier transform method with a Hanning filter. The yearly power spectra are smoothed with a running mean of 3 data points. The amplitude of solar parameters with 27-day period depends on the phase of the 11year solar cycle and is generally larger at the solar maximum. If 27-day fluctuations of solar activity affect cloud amount, we would expect the response of cloud amount to vary with the phase of the 11-year solar cycle. We then categorized the analysis period into solar maximum and minimum periods using a 1-year unit. We used the $10.7 \mathrm{~cm}$ solar radio flux
(F10.7) as a proxy of the degree of solar activity. Figure 2 shows variations in F10.7 for the period from 1980 to 2003. For the first two peaks, we define those years with smoothed F10.7 values of more than 180 (red lines) as solar maximum years (Max1 and Max2). For the third peak, the threshold is lowered to 160 (Max3). Those years with smoothed F10.7 values of less than 100 are defined as solar minimum years (Min1 and Min2). Consequently, five periods are determined according to the above procedure.

Figure $3 \mathrm{a}$ to e show the yearly power spectra of OLR for each solar activity period. The yearly spectra for solar maximum years (black lines in Fig. 3a, c, and e) are characterized by two peaks with ranges of 24-30 days (vertical red bars) and 40-60 days, except for 1991. Consequently, the two peaks are clearly evident in the average spectra (red lines). One possible reason for the lack of clear periodicity in 1991 is that incoming solar radiation in 1991 was reduced following the eruption of Mt. Pinatubo during June of that year. Therefore, the spectrum for 1991 is excluded from the average spectrum. During the solar minimum years, the range of 24-30 days is devoid of remarkable peaks (black lines in Fig. $3 b$ and d) and the spectrum features for periods of greater than $\sim 30$-day show large yearly variations, especially in terms of period.

A red (blue) line in Fig. 4a shows the power spectrum obtained by averaging all of the spectra for the maximum 


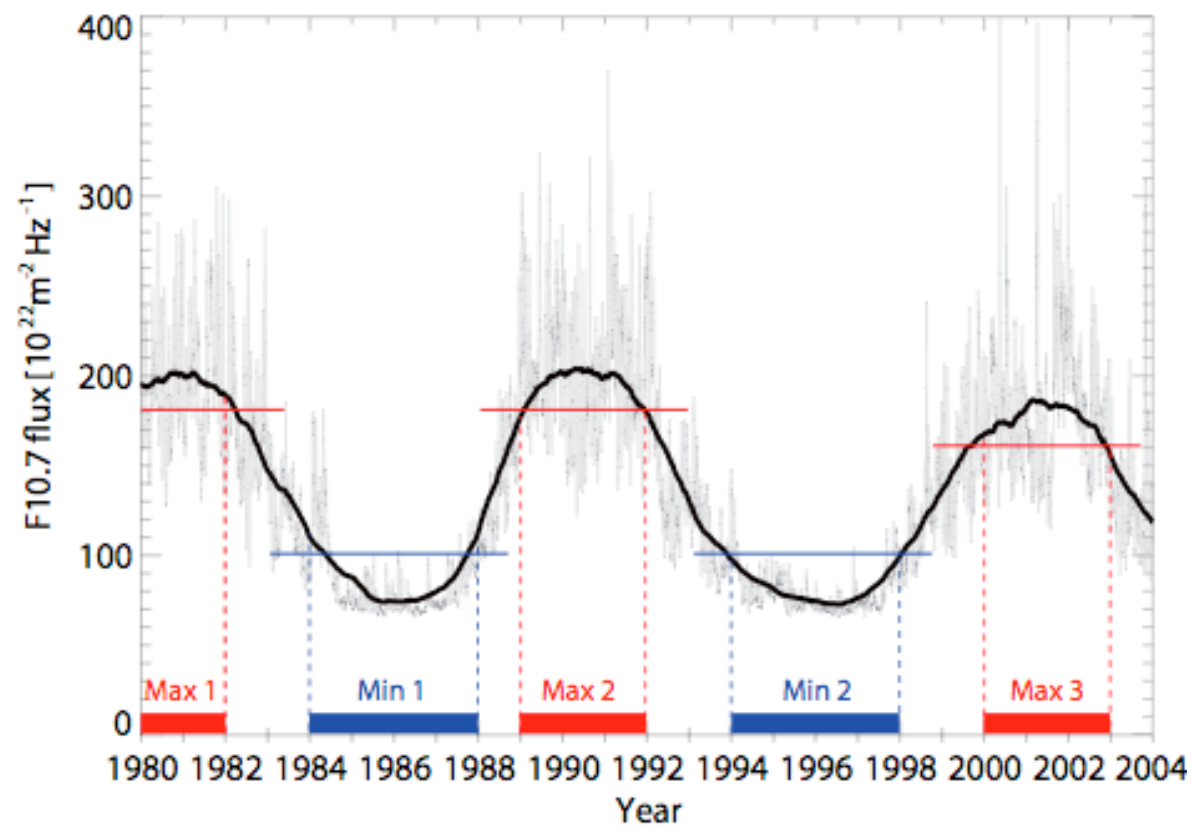

Fig. 2. Variations in F10.7 for the period from 1980 to 2003. Grey and black lines denote daily mean and 2-year running average values, respectively. Horizontal red and blue bars at the base of the figure indicate the solar maximum and minimum years categorized in this study, respectively.

(minimum) years. The spectrum for 1991 is excluded, as described above. Figure $4 \mathrm{~b}$ shows averaged spectra for F10.7 that were calculated in the same manner as those for OLR. It is evident from Fig. $4 \mathrm{~b}$ that the amplitude of solar 27day periodicity during maximum years (red line) is clearly greater than that during minimum years (blue line) by about 30 times. The shapes of the two average spectra in Fig. 4a are clearly different. Periodicities of $\sim 27$ and $\sim 50$ days are prominent in the solar maximum years (red lines), while no clear peaks appear in the range of 24-30 days in the solar minimum years (blue lines). Regarding to the error estimation for Fig. 4, we calculated the standard deviation of all years presented here as about $2\left(\mathrm{~W} / \mathrm{m}^{2}\right)$ from all the data of selected intervals in the present analysis, which suggests sufficient difference between maximum and minimum years about the peaks at $\sim 27$-day and $\sim 50$-day.

The above results can be summarized in terms of the following two new findings. First, the behaviour of variations in cloud amount depends on the phase of the 11-year solar cycle. Second, one of the two periodicities in solar maximum years has the almost same value as the cycle of solar rotation: 27 days. This suggests that the 27-day cycle in variations in solar activity controls variations in the cloud amount.

\section{Discussion}

We performed the same spectral analysis for other equatorial areas (results not shown) and found that the area in which the OLR always shows 27-day periodicity in solar maximum years is most prominent in the WPWP. This area may therefore be a kind of "accepting region" for solar activity with 27-day periodicity. Considering this fact as well as the large amplitude of 27-day periodicity, an investigation of the characteristics of WPWP may provide the key to understand the mechanism that generates the observed 27-day periodicity in cloud amount and world climate variation, as well as those of Indian Ocean.

Identification of the physical mechanisms for 27-day periodicity is not an easy task since the most solar parameters, including total solar irradiance, solar UV, and galactic cosmic ray (GCR) intensity, vary with the period of solar rotation and are modulated by the 11-year solar cycle. Therefore, we think that the mechanism determination is beyond the scope of this paper and would insist on the importance of this paper as the first fact report on 27-day periodicity in OLR. However, here, we have to mention some possible thin edges of the wedge for the explanation. Increase of GCR induced by lower solar activity could cause the increase of cloud condensation nuclei (CCN) (reviewed by Kirkby, 2007). Longer time period phenomena are sometimes successfully explained by GCR (ex. Miyahara, 2008). WPWP is one of the special places where the sea surface emits large amounts of water vapor and DMS (Dimethyl Sulfate), due to its high temperature, which are changed to $\mathrm{CCN}$ via some processes with GCR. Marsh and Svensmark (2003) indicated the high correlation between GCR and low cloud amount in WPWP for long period data. Another possible explanation 
a. $\operatorname{Max} 1(1980-1981)$

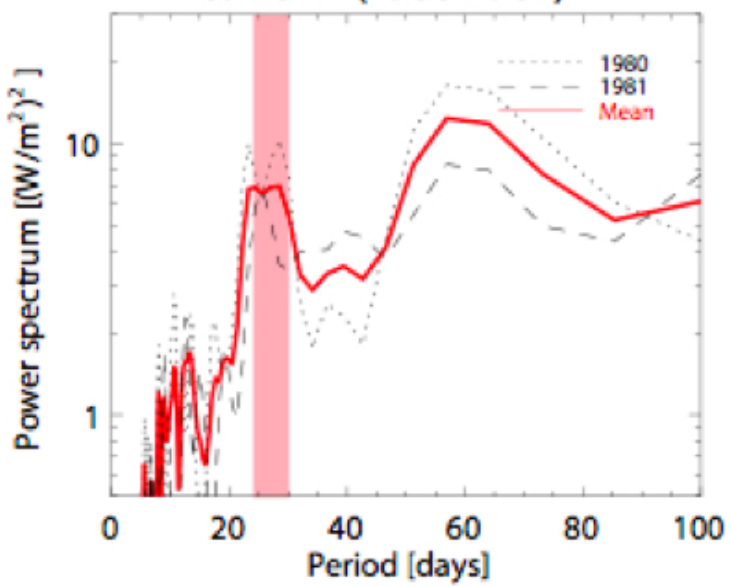

c. $\operatorname{Max} 2$ (1989-1991)
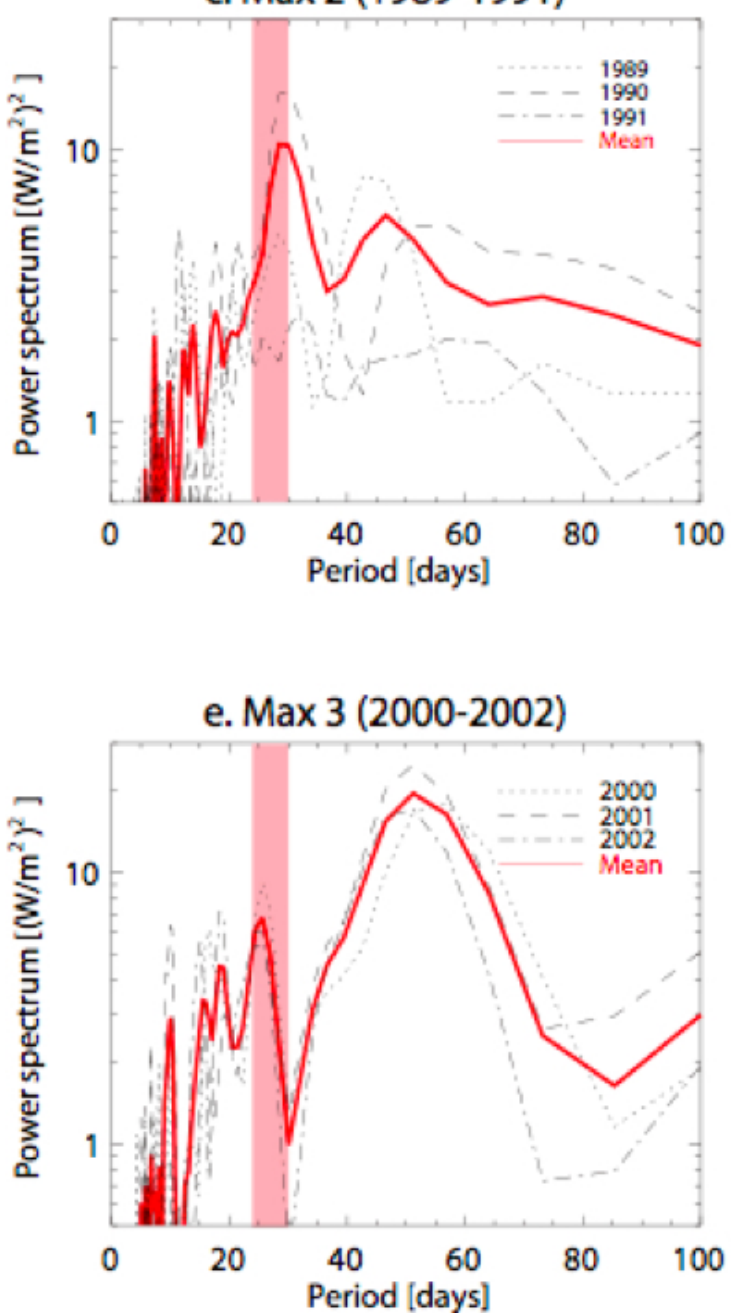
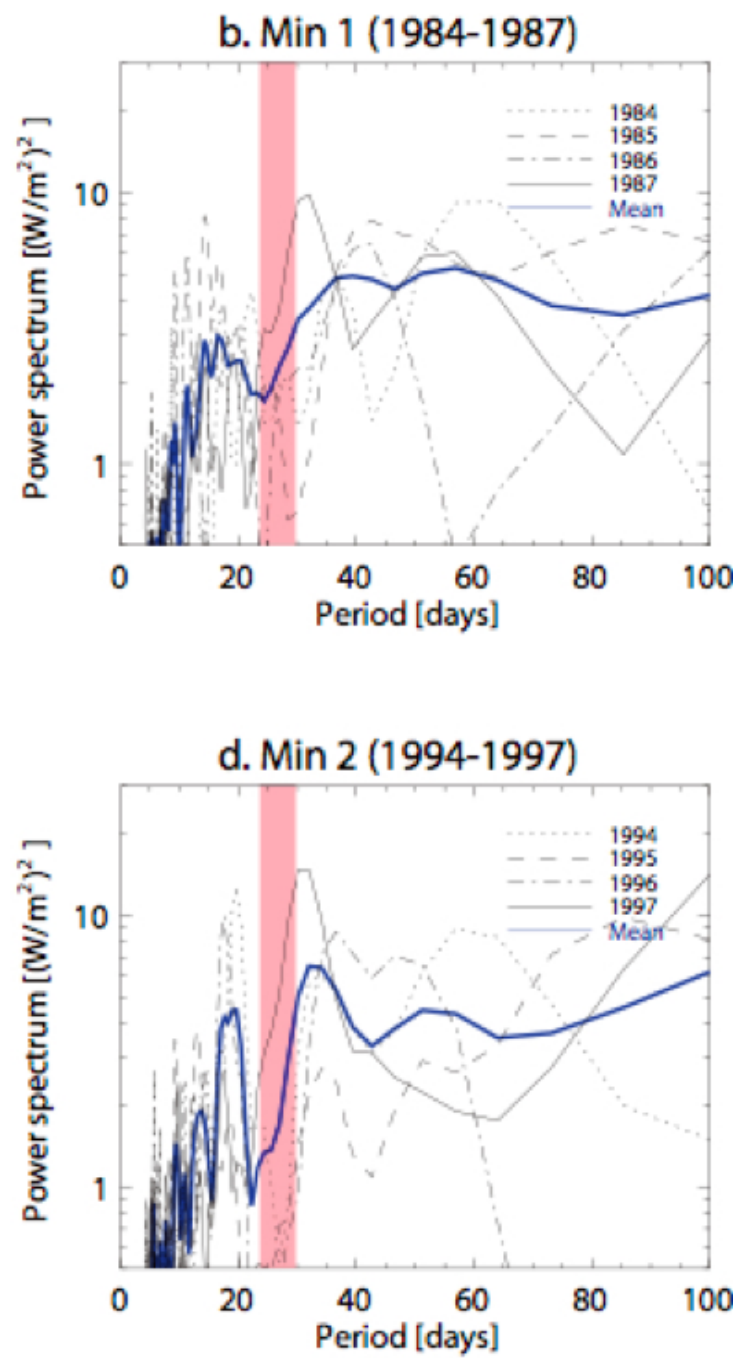

Fig. 3. Power spectra of OLR for each of the periods of solar activity. Black lines show yearly spectra. Red and blue lines represent the average spectra for the maximum and minimum periods, respectively (the spectrum for 1991 is excluded). The vertical red bars denote the period between 24 and 30 days (the solar rotation period is $\sim 27$ days). 
for the relationship between the solar parameter and cloud amount would be the mechanism related to the global circuit model, which could be modulated both by GCR and geomagnetic activities originated by solar wind variation. Modulation of the global electric circuit currents changes the distribution of highly charged aerosols, which may strongly influence cloud precipitation. This process is named as "electroscavenging" (Tinsley, 2000). WPWP is one of the most cloudy section of the sea in the world, meaning the most sensitive to the electroscavenging. At this stage we cannot exclude the effect of UV radiation via heating of the stratosphere, the effects of slight changes of total solar irradiance (TSI) and other ideas for physical connections between solar activity and cloud amount. Note again that these hypothesises should be examined quantitatively based on detailed and careful analysis, which is beyond the scope of this paper. There have been some extensive frequency analyses of the MJO already such as Whitcher et al. (2000) and suggestions for possible mechanisms for them. The review of the MJO of Madden and Julian (1994) reported on a 26-day period in the MJO for 1980-1985, and the broadband nature of the oscillation. They also report on studies which attribute the change in the period of the oscillation to warm water and El Nino. We consider that, at this moment, we cannot rule out any kinds of reasonable hypothesis for the "apparent" coincidence with solar activity. However, the intension of the present paper is to report the clear relationship between the peak around 27-day and 11-year solar activity for about 30 years, suggesting some possible linkage mechanisms. We confirmed this relationship is still valid for 2006-2008 (not shown here). In regards to lunar tide, which has almost same periodicity, we have not found any clear phase relationship to the OLR variations so far. Also we may need to consider some complicated processes from solar input to the behavior of OLR and, therefore, we believe that the next step for examination of our hypothesis is to investigate the phase relationship between OLR and other meteorological parameters in/near WPWP.

We should pay attention to the accuracies and limitations of OLR analysis. It is taken from narrowband radiances and polar-orbiting satellites, which may cause an aliasing effect due to orbital sampling of polar-orbiting satellites. However, if the 27-day period is an aliasing of shorter period variations, we need to discuss the 11-year solar activity dependence of this shorter periodicity, which may sound more unrealistic. Generally speaking, OLR is mainly modulated by high clouds and is not very sensitive to low clouds. Therefore, the result of this paper may indicate a response of high-level clouds to solar variability, in contrast to Marsh and Svensmark (2000) who suggest a link with low-level cloud amount. But we need further careful analysis in the near future.

Though the apparent periodicity in the averaged spectrum for solar minimum years (Fig. 4a) is 35 days, the periodicity shows some yearly variations in the range of 20 and 35 days. If the 27 -day periodicity in cloud amount during
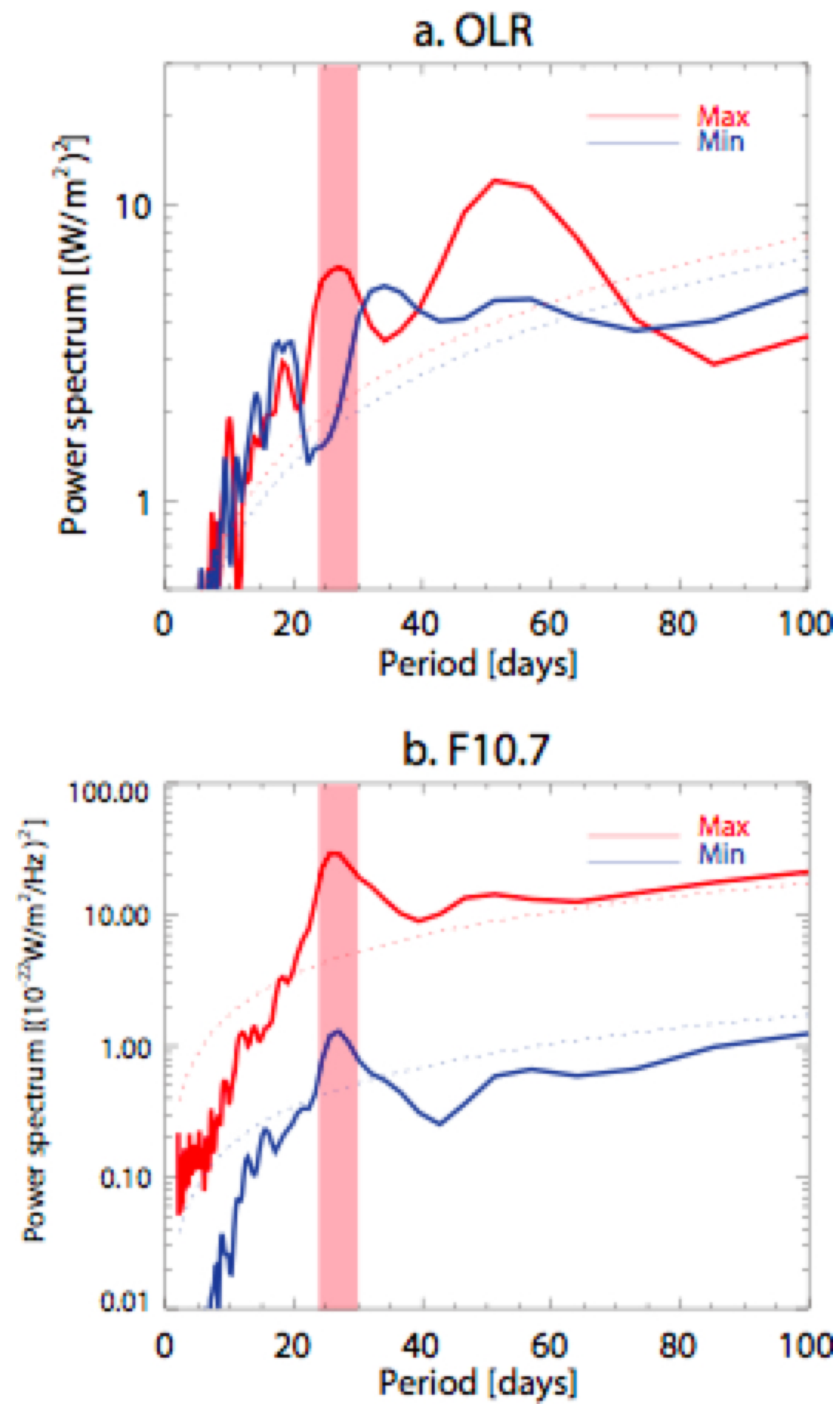

Fig. 4. (a) Averaged power spectra of OLR for the maximum (red) and minimum (blue) years. Dotted lines represent 1 -s significance levels for the two averaged spectra calculated from the standard deviation of the data. The vertical red bar represents the period between 24 and 30 days. (b) As for (a) but for F10.7.

solar maximum years is caused by solar activity, the periodicities during solar minimum years can be regarded as the internal periodicity of the Earth's atmosphere. It could be possible that the internal periodicities are synchronized with 27-day period by the weak but persistent external force caused by solar activity. The phase lag between the oscillations could provide an important hint towards the underlying physical mechanism. However, it is found that the phase relationship between the solar parameters and the OLR is not constant through out a year, namely, in a certain period of consecutive months they oscillate in the same phase while in other few months they oscillate in opposite phase, although the yearly-averaged periods are almost same: $\sim 27$ days. As 


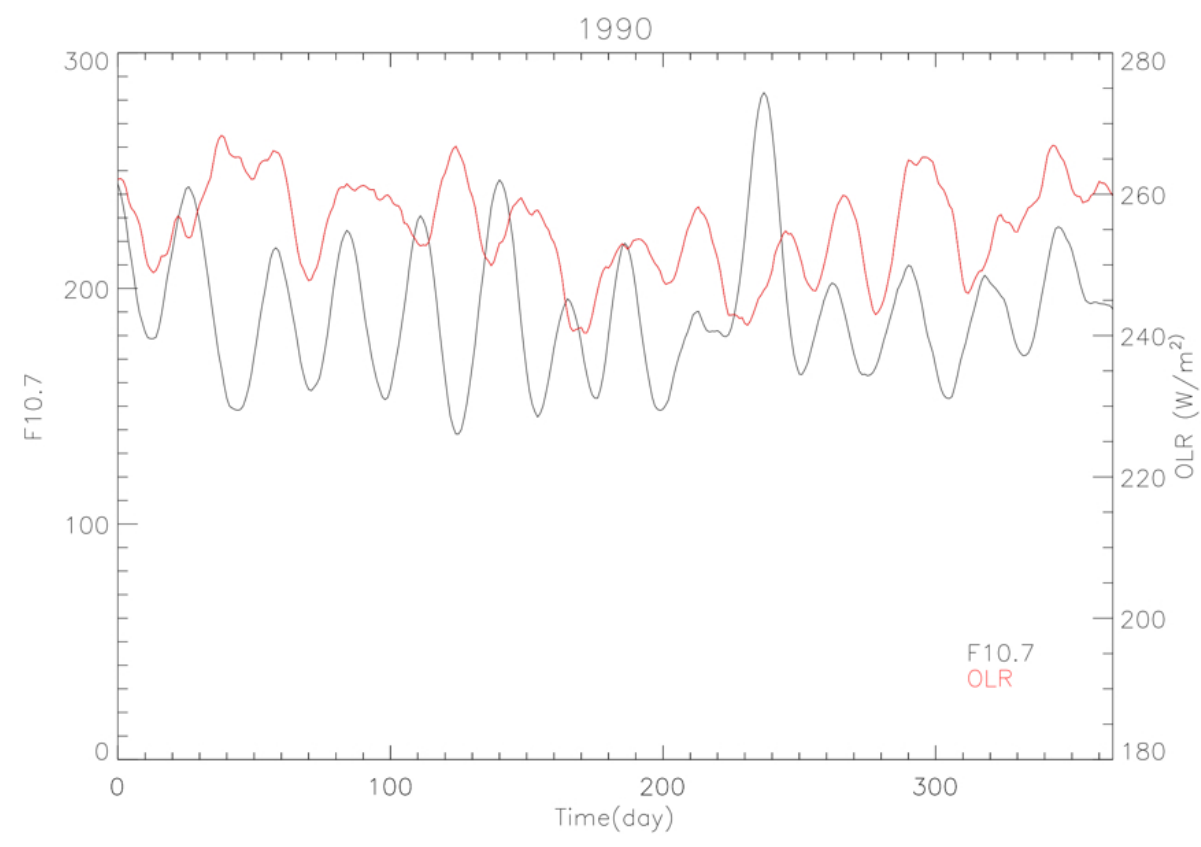

Fig. 5. Time variations of OLR in WPWP (red line) and F10.7 (black line) for 1990. The data is smoothed for 10-day running window.

one example, the temporal variation of OLR and F10.7 in 1990 is shown in Fig. 5. This fact seems to support the idea that the internal periodicity is synchronized with weak external force.

In the work by Gleisner and Thejll (2003) a significant 11year signal in the tropospheric thickness (upper and lower) for all tropical latitudes except the central and eastern Pacific was found, consistent with the present work. However, they did not find a significant response to the solar cycle in the vertical velocity in the WPWP region. This inconsistence could be attributed to the difference in spatial resolution.

The investigation of the Indian Ocean region, in which the OLR spectrum shows another maximum for $\sim 27$-day, could put some additional information into the relationships between the $\sim 27$ day OLR variations and MJO frequencies. The analysis for this region must be essential since MJO starts there. We examined this region in the same way as the WPWP and found that there sometimes exists a 27-day peak but the relationship with regards to 11-year solar activity is not so clear and constant, compared to WPWP. Also the strong enhancement as seen in Fig. 1 is caused not only by the local peak around 27-day, but sometimes caused by the enhancement over the broad range of period. In other words, Indian Ocean makes large amplitude modulations at every periods, resulting in enhancement of Fig. 1 also at about 27day. Figure 6 shows the results of FFT analysis done for Indian Ocean in the same way as Figs. 3 and 4. Still there is no doubt that the Indian Ocean is one of the most important regions to investigate 27-day periodicity of OLR, and spetial attention should be paid in future analysis.
We should be careful about the possibility of beating effect and harmonics of MJO of the longer period, which may produce an apparent peak at $\sim 27$-day. As seen in Fig. 3c, the peak in the 24-30 day range could be larger than the power in the longer period range. Also, as seen in Fig. 3a and e, no double-peaked structure is found in the MJO range, which can make a beat periodicity. The widths of MJO enhancements in Fig. 3a and e are much broader than 27-day peak, which may not cause the sharp peak around 27-day. Therefore, we think the peak at $\sim 27$-day is real phenomenon.

A periodicity of 40-60 days is also prominent and relatively stable in solar maximum years compared to minimum years. This periodicity lies within the range of the MaddenJulian Oscillation (MJO) (Madden and Julian, 1971, 1972). This result implies some direct or indirect interaction between the occurrences of the 27-day and stable MJO periodicities. However, detailed and careful analyses, paying attention to the regional and temporal variations, are required to examine this possibility.

Long-term variations in the tropospheric phenomena, including the 11-year cycle, have been generally discussed based on monthly or even yearly averaged data. In such ways, we could not find 27-day periodicity, which has much larger amplitude, up to few tens of percent, than 11-year variation of only few percent in cloud amount. The present results here suggest that we should investigate the cause of longer periodic phenomena, taking into account two possibilities: One is that the averaged solar parameter directly induces it, as has been discussed. Another is that the short-period variations of solar parameter such as 27-day 

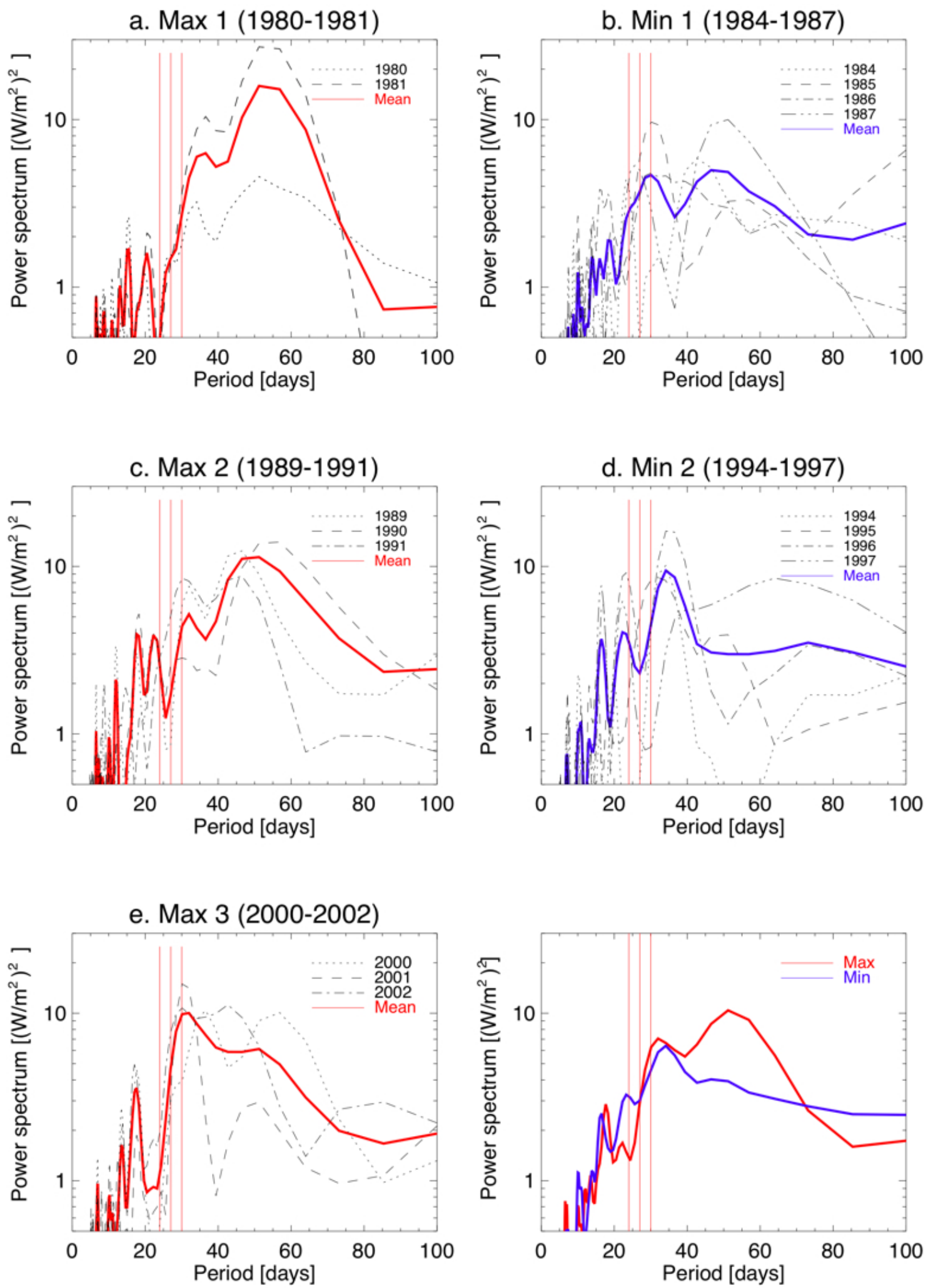

Fig. 6. Power spectra of OLR for Indian Ocean. Bottom-right is the averaged for Solar max and min periods. Format is same as Figs. 3 and 4 but without F10.7 spectrum. 
periodicity could modulate the period of climate parameter variation such as about one month variation of OLR, which has much larger amplitude in a short period, and then this modulation of period in climate parameter induces the longer period variation, as a structure of the time hierarchy.

\section{Conclusions}

We made simple frequency analysis for cloud amount based on OLR data of 25 years, focusing on the relationship between short-term $(\sim 1$ month) changes and the 11-year solar cycle. It was found that the $\sim 27$-day variation of OLR is prominent in WPWP in solar maximum years. This fact suggests a possibility that the solar rotation influences the variations of the cloud amount in that area. Taking into account the fact that the apparent periodicity of cloud amount in the averaged spectrum for solar minimum years exists at $\sim 35$ days, which is clearly longer than 27 days, the possibility of synchronization with a weak external source should be examined. The averaged spectrum of OLR variation in solar maximum years also shows a significant enhancement in the period range of 40-60 days, which corresponds to MJO periods, indicating some possible connection between them. Though some physical mechanisms to explain the solar activity effect on cloud amount variation could be proposed, careful and multidirectional approaches are strongly desired in the near future.

Acknowledgements. The interpolated OLR data were provided by the NOAA/OAR/ESRL PSD. The F10.7 data were acquired by the Dominion Radio Astrophysical Observatory. This work was supported by Grant-in-aid for scientific research No. 119002002.

Edited by: J. Curtius

\section{References}

Damon, P. E. and Laut, P.: Pattern of strange errors plagues solar activity and terrestrial climate data, Eos Trans. AGU, 85(39), doi:10.1029/2004EO390005, 2004.

Gleisner, H. and Thejll, P.: Patterns of tropospheric response to solar variability, Geophys. Res. Lett., 30(13), 1711, doi:10.1029/2003GL017129, 2003.
Haigh, J. D.: The role of stratospheric ozone in modulating the solar radiative forcing of climate, Nature, 370, 544-546, 1994.

Hood, L. L.: The temporal behaviour of upper stratospheric ozone at low latitudes - Evidence from Nimbus 4 BUV data for shortterm responses to solar ultraviolet variability, J. Geophys. Res. 89, 9557-9568, 1984.

Kirkby, J.: Cosmic rays and climate, Surv. Geophys., 28, 333-375, doi:10.1007/s10712-008-9030-6, 2007.

Kodera, K.: On the origin and nature of the interannual variability of the winter stratospheric circulation in the northern hemisphere, J. Geophys. Res., 100, 14077-14087, 1995.

Liebmann, B. and Smith, C. A.: Description of a complete (interpolated) outgoing longwave radiation dataset, B. Am. Meteor. Soc., 77, 1275-1277, 1996.

Madden, R. A. and Julian, P. R.: Detection of a 40-50 day oscillation in the zonal wind in the tropical Pacific, J. Atmos. Sci., 28, 702-708, 1971.

Madden, R. A. and Julian, P. R.: Description of global-scale circulation cells in the tropics with a 40-50 day period, J. Atmos. Sci. 29, 1109-1120, 1972.

Madden, R. A., and Julian, P. R.: Observations of the 40-50-Day Tropical Oscillation, Mon. Weather Rev., 122, 814-837, 1994.

Marsh, N. and Svensmark, H.: Galactic cosmic ray and El Niño Southern Oscillation trends in International Satellite Cloud Climatology Project D2 low-cloud properties, J. Geophys. Res., 108(D6), 4195, doi:10.1029/2001JD001264, 2003.

Miyahara, H., Yokoyama, Y., and Masuda, K.: Possible link between multi-decadal climate cycles and periodic reversals of solar magnetic field polarity, Earth Planet. Sci. Lett., 272, 290-295, 2008.

Nastrom, G. D. and Belmont, A. D.: Preliminary results on 27-day solar rotation variation in stratospheric zonal winds, Geophys. Res. Lett. 5, 665-668, 1978.

Shindell, D., Rind, D., Balachandran, N., Lean, J., and Lonergan, P.: Solar cycle variability, ozone, and climate, Science, 284, 305308, 1999.

Svensmark, H.: Influence of cosmic rays on Earth's climate, Phys. Rev. Lett., 81, 5027-5030, 1998.

Tinsley, B. A., Rohrbaugh, R. P., Hei, M., and Beard, K. V.: Effects of image charges on the scavenging of aerosol particles by cloud droplets and on droplet charging and possible ice nucleation processes, Atmos. Res., 57, 2118-2134, 2000.

Whitcher, B., Guttorp, P., and Percival, D. B.: Wavelet analysis of covariance with application to atmospheric time series, J. Geophys. Res.-Atmos., 105(D11), 14941-14962, 2000. 